\title{
Inter-band Carrier Aggregation in Heterogeneous Networks: Design and Assessment
}

\author{
Georgia D. Ntouni*†, Alexandros-Apostolos A. Boulogeorgos*†, Dimitrios S. Karas ${ }^{\dagger}$, \\ Theodoros A. Tsiftsis ${ }^{* \ddagger}$, Fotis Foukalas*, Vasileios M. Kapinas ${ }^{\dagger}$ and George K. Karagiannidis ${ }^{\dagger}$ \\ *Industrial Systems Institute, Athena RC, Rion Patras, 26504, Greece \\ Email: foukalas@isi.gr \\ ${ }^{\dagger}$ Department of Electrical and Computer Engineering, Aristotle University of Thessaloniki, Greece \\ Email: \{gntouni, ampoulog, dkaras, kapinas, geokarag\}@auth.gr \\ ${ }^{\ddagger}$ Department of Electrical Engineering, Technological Educational Institute of Central Greece, Greece \\ Email: tsiftsis@teilam.gr
}

\begin{abstract}
This paper deals with the performance assessment of the Long Term Evolution (LTE)-Advanced Release 12 physical downlink channel, emphasizing on the Carrier Aggregation (CA) technology and its recent advances, such as the challenging interband non-contiguous solution. By processing the LTE-Advanced waveforms in the time domain (instead of the more common baseband), we describe the underlying system model and the associated simulation setup in detail. The error performance of the system is evaluated under different physical layer parameters and CA scenarios, according to the latest updates of the Third Generation Partnership Project (3GPP) technical specifications. Our analysis reveals that the Heterogeneous Band (HetBand) non-contiguous CA technology can be efficiently applied to the design of next generation mobile broadband networks, given that the exploitation of both unlicensed and frequency dispersed bands might be a promising solution against the spectrum scarcity.

Index Terms-Carrier aggregation, inter-band spectrum aggregation, heterogeneous bands, long term evolution-advanced
\end{abstract}

\section{INTRODUCTION}

Mobile broadband communications experience today great success with major standardization bodies, such as the Third Generation Partnership Project (3GPP), leading a continuous effort for the definition of the next-generation Radio Access Technologies (RAT)s. In October 2010, the Long Term Evolution (LTE)-Advanced (a.k.a. LTE Release 10 \& Beyond) has been approved by the International Telecommunication Union (ITU) as a real fourth generation (4G) wireless technology and as such it has been part of the International Mobile Telecommunications (IMT)-Advanced family of radio interfaces [1].

In the meantime, the major standardization bodies and key industry players are organizing a time frame to define a fifth generation $(5 \mathrm{G})$ for wireless communications technology, which is expected to be between 2016 and 2018, followed by initial deployments around 2020 [2]. In the transition period from $4 \mathrm{G}$ to $5 \mathrm{G}$, which is also called the $5 \mathrm{G}$ Era, we will see a shift towards network efficiency with systems based on dense

This work was carried out and funded within the framework of SOLDER ICT-619687 FP7 STREP project (2013-2016).

978-1-4799-5863-4/14/\$31.00 (C) 2014 IEEE
Heterogeneous Network (HetNet) architectures, and increased use of the radio spectrum through spectral aggregation techniques [3], [4]. These two technology trends form the basis of the network densification over space and frequency [4].

Particularly, LTE-Advanced systems are expected to support high data rates after the Carrier Aggregation (CA) technology was initially introduced as part of Release 10 . This means of extending the transmission bandwidth is considered one of the most highly anticipated features of LTE-Advanced because it allows the system to aggregate up to five carriers of bandwidth $20 \mathrm{MHz}$ each, providing a total bandwidth of $100 \mathrm{MHz}$. Within such a wide band, data rates as high as 1 Gbps for the downlink and $500 \mathrm{Mbps}$ for the uplink can be achieved. By aggregating non-contiguous carriers, fragmented spectrum can be more efficiently utilized. Additional advantages are offered by $\mathrm{CA}$ in terms of spectrum efficiency, deployment flexibility, backward compatibility, and more [5].

Various deployment scenarios for homogeneous and heterogeneous networks are supported by continuous and noncontiguous CA with proper utilization of different component carriers (CC)s. Several overview papers on CA exist in the literature [5]-[8], while others deal with the performance assessment of this technique under sporadic aggregation scenarios [9]-[11]. In this paper, the three 3GPP specified CA options, as well as basic deployment scenarios of CA in HetNets are discussed. We proceed with the simulation setup and the associated system model for the LTE-Advanced Release 12 physical downlink channel, assuming $\mathrm{CA}$ and processing the associated waveforms in the time domain. Finally, the performance of representative CA scenarios in terms of error probability is investigated under different values of critical physical layer (PHY) parameters, such as the total aggregated bandwidth, the channel conditions, and the encoding scheme.

\section{Carrier Aggregation Options in LTE-Advanced}

Generally, CA systems are intended to improve data rates for users within overlapped areas of cells by allowing the operators to deploy a system with extended bandwidth. This 


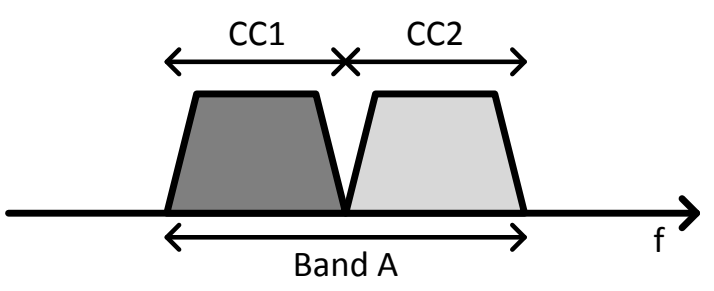

Intra-band contiguous CA

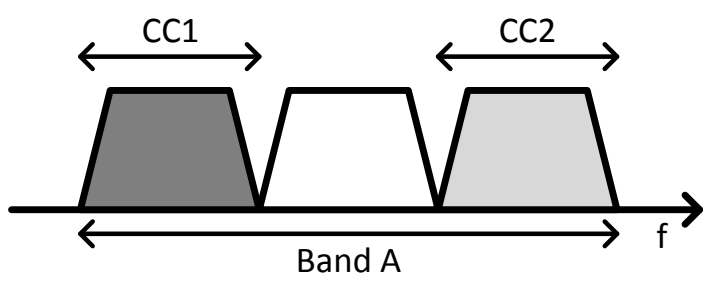

Intra-band non-contiguous $\mathrm{CA}$

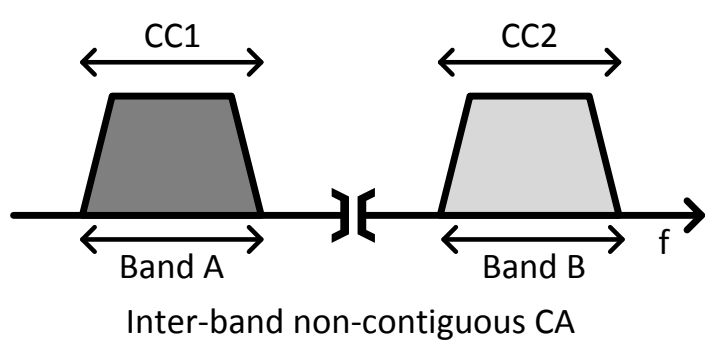

Fig. 1. The specified CA scenarios for LTE-Advanced.

can be implemented by aggregating several smaller CCs, while providing backward compatibility to legacy users [12].

In order to achieve backward compatibility and allow the transmission of data to one user using both the newly deployed LTE networks and the evolving networks of previous technologies, such as the High-Speed Packet Access (HSPA) and the Global System for Mobile (GSM) communications, the Evolved Universal Terrestrial Radio Access (E-UTRA) frequency bands that can be aggregated support flexible channel bandwidths in both the uplink and downlink, i.e., 1.4, 3, 5, 10, 15 and $20 \mathrm{MHz}$.

As shown in Fig. 1, there are three different CA scenarios, which have been proposed by 3GPP [13]:

1) Intra-band contiguous $C A$ : A single frequency band is utilized and all CCs used are adjacent to each other. Although this may be a less likely scenario given the frequency allocation today, it can be common when new spectrum bands like $3.5 \mathrm{GHz}$ will be allocated in the future in various parts of the world. The spacing between the center frequencies of the contiguously aggregated CCs is a multiple of $300 \mathrm{KHz}$ so as to be compatible with the $100 \mathrm{KHz}$ frequency raster of Release 8/9 and preserving orthogonality of the subcarriers with $15 \mathrm{KHz}$ spacing [6].

2) Intra-band non-contiguous $C A$ : A single frequency band is utilized but the CCs used are not adjacent to each

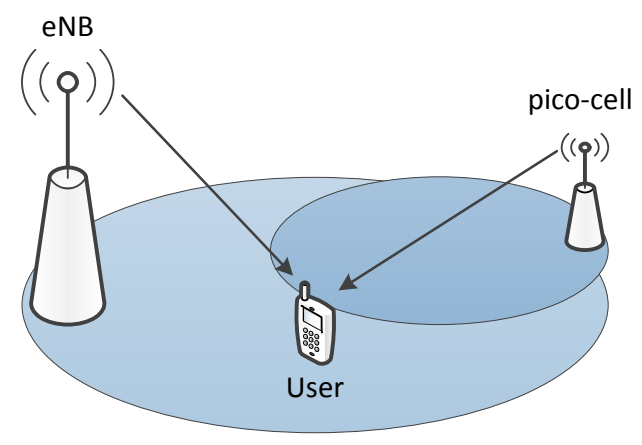

Fig. 2. Carrier aggregation over Heterogeneous Bands (HetBand)s utilized by an LTE-Advanced BS and a pico-cell.

other, i.e., the CCs are far from each other. This adds significant complexity, especially to the User Equipment (UE), where space, power and cost are major considerations.

3) Inter-band non-contiguous CA: The CCs used belong to different frequency bands, and as such they are far from each other. This scenario is very promising for future high data-rate mobile communications due to the inevitable fragmentation of bands, some of which are only $10 \mathrm{MHz}$ wide. Concerning the UE, it requires the use of multiple transceivers within the single item, thus introducing new challenges related to cost, performance and power consumption. Additionally, there are further complexities resulting from the necessity to reduce intermodulation and cross modulation among the individual transceivers. With this type of aggregation, mobility robustness can potentially be improved by exploiting radio propagation characteristics of different bands [14].

In all cases, multiple CCs are aggregated to serve a single LTE-Advanced UE unit. Regarding the UE cost, complexity, and power consumption, it is easier to implement continuous CA without making many changes to the PHY structure of existing LTE systems. It is possible to use a single Fast Fourier Transform (FFT) module and a single radio frequency component to achieve continuous CA for the LTE-Advanced UE unit, while providing backward compatibility to LTE systems. Compared to non-continuous $\mathrm{CA}$, it is easier to implement resource allocation and management algorithms for continuous CA [7]. However, due to the fact that the spectrums currently allocated are scattered and a continuous $100 \mathrm{MHz}$ bandwidth is unlikely to be available for LTE-Advanced system, the noncontiguous CA approach seems to be more practical.

\section{Scenarios of CARrier Aggregation in HetNets}

According to [15], the CA deployment scenarios for two $\mathrm{CCs}$ at frequencies $F_{1}$ and $F_{2}\left(F_{2}>F_{1}\right)$ are:

- Deployment scenario 1: Cells with carrier frequencies of $F_{1}$ and $F_{2}$ are overlaid with $F_{1}$ and $F_{2}$ in the same band. In this case, almost the same coverage is provided on both carriers due to the similar path loss within the same band. 
TABLE I

OFDM PARAMETERS

\begin{tabular}{|c|c|c|c|c|c|c|c|}
\hline Bandwidth (MHz) & 1.4 & 3 & 5 & 10 & 15 & 20 \\
\hline$\Delta f$ & \multicolumn{7}{|c|}{$15 \mathrm{KHz}$} \\
\hline$N$ & 128 & 256 & 512 & 1024 & 1536 & 2048 \\
\hline$N_{s c}$ & 72 & 180 & 300 & 600 & 900 & 1200 \\
\hline$N_{c p}$ & 9 & 18 & 36 & 72 & 108 & 144 \\
\hline$f_{s}(\mathrm{MHz})$ & 1.92 & 3.84 & 7.68 & 15.36 & 23.04 & 30.72 \\
\hline
\end{tabular}

- Deployment scenario 2: Cells with carrier frequencies $F_{1}$ and $F_{2}$ are overlaid, where $F_{1}$ and $F_{2}$ are in different bands. Unlike the scenario 1 , the coverage of each frequency is different.

- Deployment scenario 3: Antennas in cells with frequency $F_{2}$ are directed to the cell boundaries with frequency $F_{1}$ to improve the cell edge data rate and throughput.

- Deployment scenario 4: Cells with frequency $F_{1}$ provide macro coverage, and remote radio heads with frequency $F_{2}$ are used to improve throughput at hot spots.

- Deployment scenario 5: Similar to deployment scenario 2. Frequency selective repeaters are additionally deployed.

Initially, CA will only be used to aggregate carriers on the same site. However, a centralized architecture allows also for multi-site CA [16]. LTE-Advance Release 11 introduced the support of multi-site CA by supporting multiple uplink timing advance enhanced uplink power control for HetNets [17]. In this case, the macro base station with full coverage could serve as primary cell, providing system information, control signalling, and bandwidth limited data transmission, while the small base station would serve local high data rate requirements. This $\mathrm{CA}$ technique will also be beneficial when macro- and pico-cells are using dedicated carriers [18], as illustrated in Fig. 2. An alternative deployment is when the macro- and the pico-cells operate with own control signalling on both frequency layers. Such deployment will require advanced interference management.

\section{System model and Simulation Setup Details}

The downlink PHY connection of our system is illustrated as the block diagram in Fig. 3. This setup emulates the fundamental processes involved in the downlink transmission of the LTE-Advanced, according to the Release 12 PHY specifications developed by 3GPP [19].

The information bits are initially encoded by the Turbo encoder with a data rate equal to $1 / 3$. LTE-Advanced supports a variety of coding and modulation schemes, which can be flexibly modified in order to adapt to the instantaneous channel and interference conditions realized by each user. The encoded data stream is then modulated with one out of the possible modulation schemes supported by LTE-Advanced, namely QPSK, 16-QAM and 64-QAM.

Orthogonal Frequency-Division Multiplexing (OFDM) was adopted in LTE-Advanced since it is a promising tool for combating the Inter-Symbol Interference (ISI) caused by a frequency-selective fading channel. This is achieved by dividing the broadband channel into a set of parallel narrowband (and hence flat) subchannels, i.e., by demultiplexing the data sequence into several streams transmitted in parallel on different subcarriers and with sufficiently small symbol rates (with respect to the channel coherence bandwidth) to consider the subchannels as flat [20]. In our case, the spacing $\Delta f$ between the OFDM subcarriers is specified and fixed to 15 KHz. The rest OFDM parameters, namely the FFT/IFFT size $N$, the number of useful subcarriers $N_{s c}$, the Cyclic Prefix (CP) length $N_{c p}$, and the sampling frequency $f_{s}$, are selected from Table I depending on the bandwidth of the $\mathrm{CC}$ in the frequency band utilized. We recall here that the $\mathrm{CP}$ is specified in terms of samples and is added to the OFDM symbol after the IFFT in order to mitigate the ISI effects.

The OFDM symbols are then converted from digital samples to an analog waveform, or equivalently, the signal is transformed from a dicrete-time domain to a continuous-time domain representation. In order to produce the continuoustime signal, a transmit filter is first applied to the complex samples of each subcarrier followed by the appropriate digitalto-analog (D/A) filter. In our simulations, we have employed Butterworth D/A and reconstruction filters of appropriate order and cut-off frequency, depending on the simulated scenario.

In order to be transmitted, the baseband analog signal $s_{b}(t)$ (produced by the D/A conversion) shall be translated to a higher frequency band of center frequency equal to $f_{c}$, which is selected from those specified by $3 \mathrm{GPP}$, using the formula

$$
s_{u p}(t)=s_{b}(t) e^{j 2 \pi f_{c} t} .
$$

Although CA of up to five CCs has been proposed by 3GPP, the current specifications include CA scenarios of only two CCs [13]. Therefore, being consistent with the state-of-the-art standardization procedures, we consider two analog signals that are simultaneously transmitted towards a single user. The high center frequencies involved in the upconvertion process belong to the already specified frequency bands allowed to be paired, having appropriate bandwidths that comply with the 3 GPP requirements.

The implementation of the CA technique, given the upconverted analog signals, involves adding them up and transmitting the produced signal to the user. Assuming that $f_{c 1}$ and $f_{c 2}$ are the two center frequencies of the individual bands, the aggregated signal can be simply given by the formula

$$
s_{C A}(t)=s_{t b 1}(t) e^{j 2 \pi f_{c 1} t}+s_{t b 2}(t) e^{j 2 \pi f_{c 2} t},
$$

where $s_{t b 1}(t)$ and $s_{t b 2}(t)$ are the time-domain transmitted baseband signals, associated with the bands to be aggregated. Without loss of generality, the channel between the transmitter and the receiver is modeled as flat fading Rayleigh in the presence of Additive White Gaussian Noise (AWGN).

In the receiver side, the first process taking place is the separation of the two aggregated signals. Thus, after downcon- 


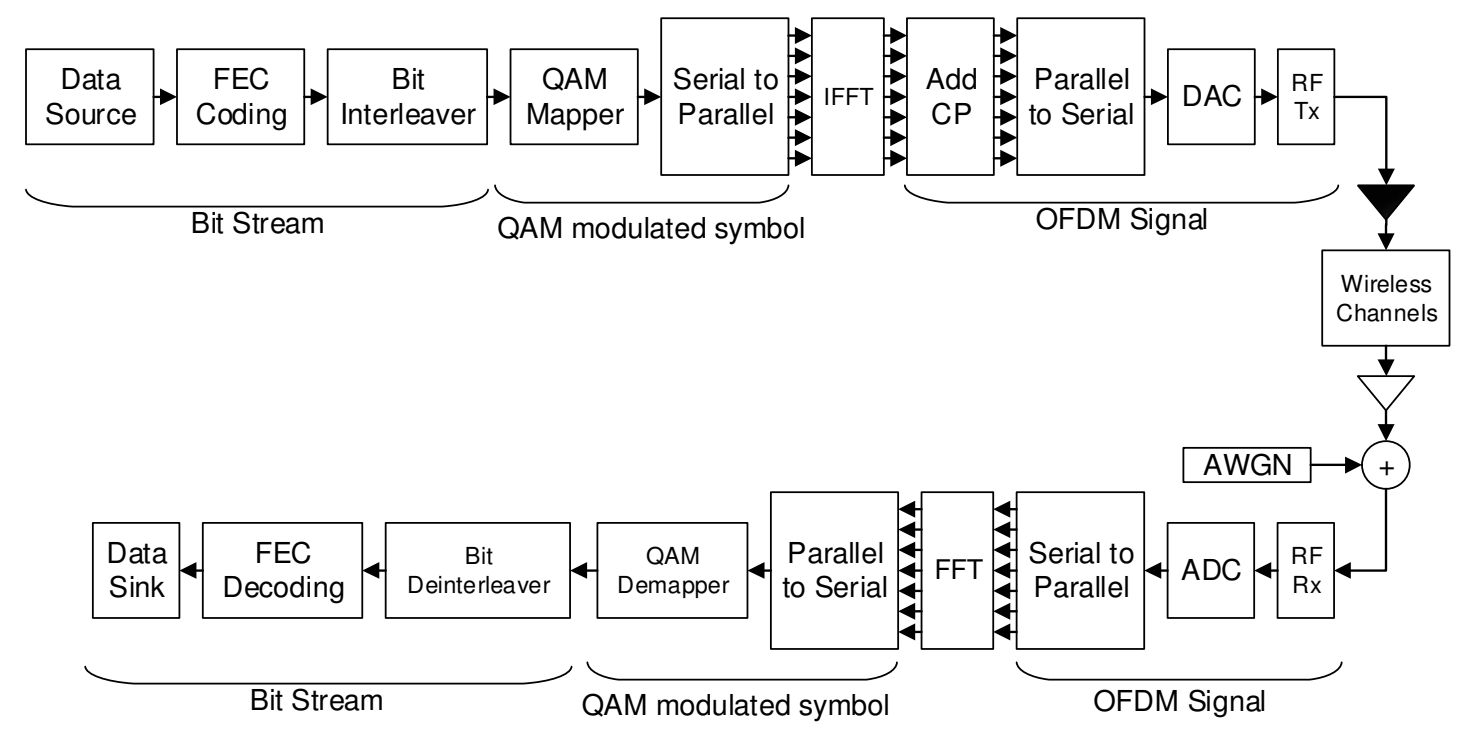

Fig. 3. Downlink physical layer block diagram of an LTE-Advanced Release 12 system.

TABLE II

INTER-BAND NON-CONTIGUOUS CA SCENARIOS.

\begin{tabular}{|c|c|c|c|}
\hline $\begin{array}{c}\text { E-UTRA } \\
\text { CA Band }\end{array}$ & $\begin{array}{c}\text { E-UTRA } \\
\text { Frequency Band }\end{array}$ & $\begin{array}{c}f_{\text {low }}-f_{\text {high }} \\
(\mathrm{MHz})\end{array}$ & $\begin{array}{c}\text { Bandwidth } \\
(\mathrm{MHz})\end{array}$ \\
\hline \multirow{2}{*}{$\mathrm{CA}(1,5)$} & 1 & $2110-2170$ & 10 \\
\cline { 2 - 4 } & 5 & $869-894$ & 10 \\
\hline \multirow{2}{*}{$\mathrm{CA}(3,7)$} & 3 & $1805-1880$ & 10,15 or 20 \\
\cline { 2 - 4 } & 7 & $2620-2690$ & 10,15 or 20 \\
\hline
\end{tabular}

version, the signals $s_{r b 1}(t)$ and $s_{r b 1}(t)$ at the receiver, become

$$
\begin{aligned}
& s_{r b 1}(t)=s_{C A}(t) e^{-j 2 \pi f_{c 1} t} \\
& s_{r b 2}(t)=s_{C A}(t) e^{-j 2 \pi f_{c 2} t} .
\end{aligned}
$$

The rest of the reception process is straightforward, since it is just the reverse of the one having taken place at the transmitter, as described above. Finally, it should be noted that the filtering operations, both at the transmitter and the receiver, produce delay. Since OFDM is sensitive to timing offsets, the time delay is estimated and considered at the filtered signals.

Based on the system model described above, we simulate all three different CA options mentioned in Section II. Concerning the intra-band (either contiguous or non-contiguous) CA scenarios, the CCs considered are taken from the LTE frequency band 7 , as these are specified in the latest 3GPP specifications. Besides, one more CA scenario, that is not yet officially specified, is simulated in order to evaluate the performance of the CA technique over dispersed frequency bands. To be more precise, the $\mathrm{CA}(1,5)$ scenario is compared with the one involving aggregation of one $\mathrm{CC}$ at $900 \mathrm{MHz}$ along with one at $5 \mathrm{GHz}$.

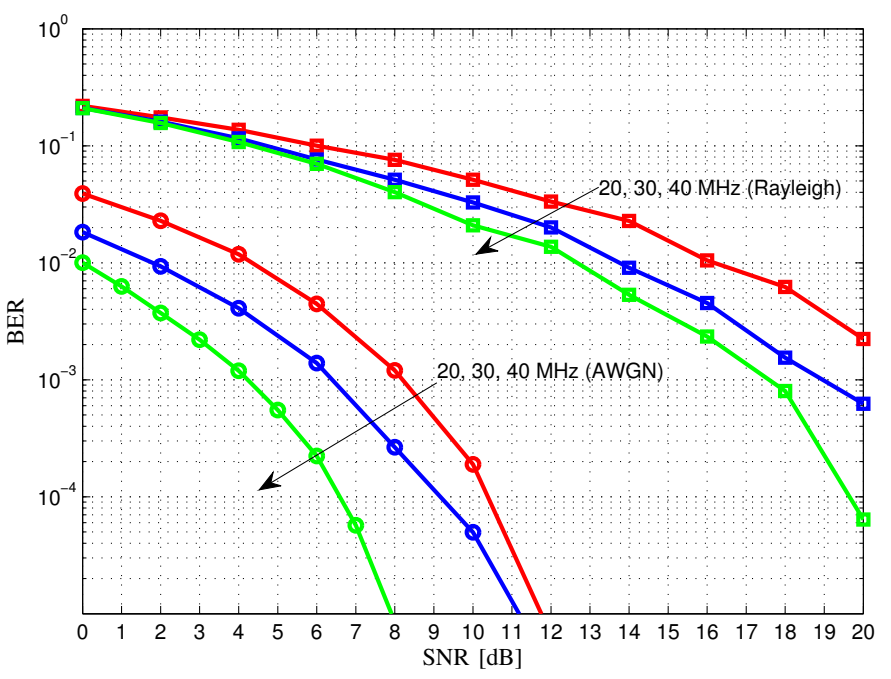

Fig. 4. Performance of inter-band non-contiguous $\mathrm{CA}(3,7)$ scenario with 20 , 30 , and $40 \mathrm{MHz}$ total bandwidths, over two extreme channel conditions.

\section{COMPARISON OF DifFERENT CA ScEnARIOS}

In this section, we simulate the inter-band non-contiguous spectrum aggregation scenarios defined in the 3GPP Release $10 \mathrm{CA}$ specifications [13], which have been reproduced in Table II for the sake of clarity. Furthermore, intra-band CA scenarios and new aggregation options utilizing bands in the unlicensed spectrum have been simulated. It is important to note here that the filters employed are all Butterworth of the same order in order to verify that their quality factor does not influence the simulation results. All CA scenarios are simulated in terms of the Bit-Error Rate (BER) performance for the two cases of a Single-Input Single-Output (SISO) flat fading Rayleigh and a pure AWGN channel. Finally, in order to reduce the induced computation penalty, the (Turbo) 


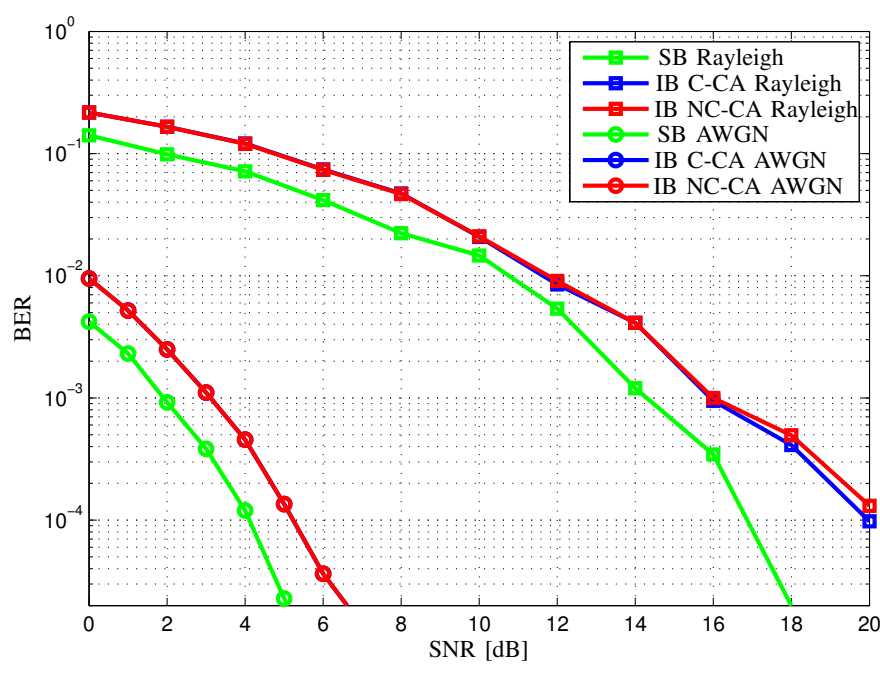

Fig. 5. Comparison of intra-band Contiguous (C)- or Non-Contiguous (NC)CA scenarios using the LTE-Advanced band 7 with $20 \mathrm{MHz}$ total bandwidth.

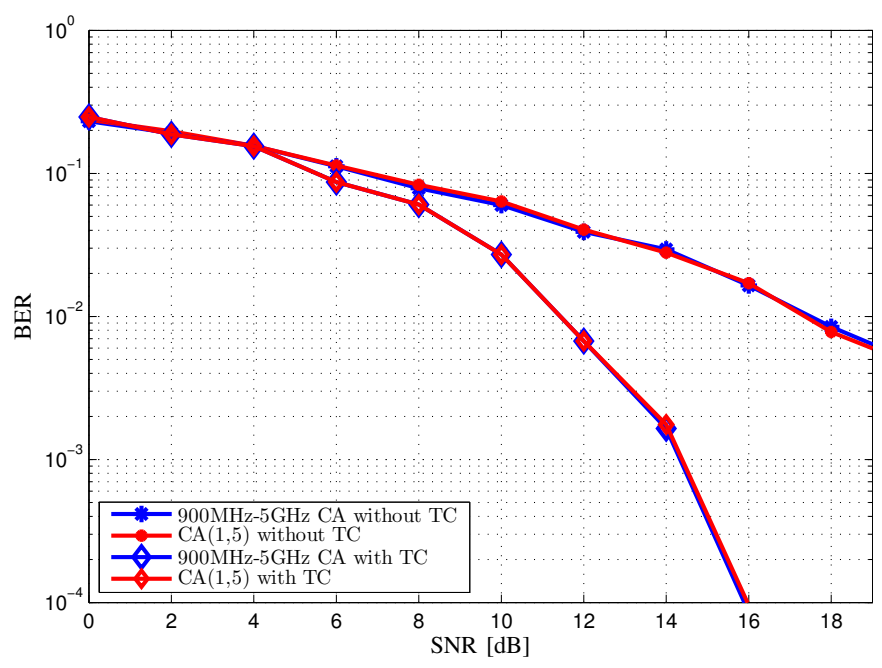

Fig. 6. Performance of two inter-band CA scenarios over dispersed bands i) licensed $\mathrm{CA}(1,5)$ and ii) unlicensed $900 \mathrm{MHz}-5 \mathrm{GHz}$, with or without Turbo Coding (TC) and total bandwidth in both cases equal to $20 \mathrm{MHz}$.

encoding process is considered only in one particulary case; the $\mathrm{CA}(1,5)$ scenario over dispersed (licensed or not) bands.

Firstly, in Fig. 4, the BER performance of the $\mathrm{CA}(3,7)$ scenario is given for three different (eligible) values of the total bandwidth, namely $20 \mathrm{MHz}, 30 \mathrm{MHz}$ and $40 \mathrm{MHz}$, assuming both flat fading Rayleigh and AWGN channel conditions. Not surprisingly, the illustrated curves reveal that the more the total available bandwidth, the better the performance in terms of BER becomes. Obviously, the best results are provided for the combination of $40 \mathrm{MHz}$ total bandwidth along with the presence of (the ideal) AWGN channel conditions.

In Fig. 5, the BER performance of the two intra-band CA options (discussed in Section II) applied over the LTEAdvanced frequency band 7 is compared under both flat fading Rayleigh and AWGN channel conditions, assuming in all cases a total bandwidth of $20 \mathrm{MHz}$. In more detail, the intra-band
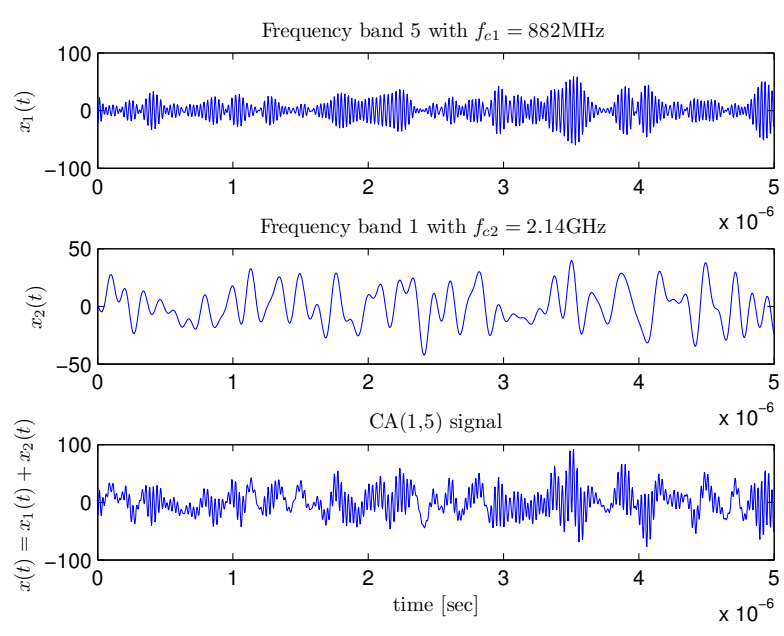

Fig. 7. Time-domain signal waveforms before and after the CA operation assuming the $\mathrm{CA}(1,5)$ scenario with total bandwidth equal to $20 \mathrm{MHz}$.

contiguous aggregation of the two $10 \mathrm{MHz}$ bands $2649 \mathrm{MHz}$ and $2661 \mathrm{MHz}$ (both belonging in the LTE-Advanced band 7 ) is considered. Its performance is compared to the intraband non-contiguous CA of the frequency bands $2630 \mathrm{MHz}$ and $2680 \mathrm{MHz}$, each of $10 \mathrm{MHz}$ bandwidth (both belonging in the LTE-Advanced band 7 too). It can be observed that the center frequencies of the two contiguously aggregated CCs have a spacing which is a multiple of $300 \mathrm{KHz}$ to comply with the specifications. Additionally, the performance of a system utilizing a Single Band (SB) with center frequency $2655 \mathrm{MHz}$ and bandwidth $20 \mathrm{MHz}$ is provided as a benchmark. Interestingly, we can notice that the two intraband CA options give the same BER performance, though under different hardware complexity requirements (consider the implementation challenges in the design of filters with center frequencies which are very close one to another).

In Fig. 6, two HetBand non-contiguous CA scenarios of dispersed frequency bands, namely the $\mathrm{CA}(1,5)$ and the aggregation of the $900 \mathrm{MHz}-5 \mathrm{GHz}$ bands, are simulated in terms of the BER performance for the case of a SISO flat fading Rayleigh channel with total bandwidth $20 \mathrm{MHz}$. For illustration purposes, we consider both Turbo coded and uncoded transmission sequences in the same plot. It shall be noted here that the Unlicensed National Information Infrastructure (U-NII) bands in $5 \mathrm{GHz}$ are used by the IEEE $802.11 \mathrm{a} / \mathrm{n}$ technologies of the Wireless Local Area Networks (WLAN) systems and also as an unlicensed LTE-Advanced frequency band. We observe that both HetBand CA scenarios provide the same performance, though not taking into account the hardware complexity requirements for each case.

Finally, for the $\mathrm{CA}(1,5)$ scenario with total aggregated bandwidth equal to $20 \mathrm{MHz}$, the transmitted signal waveforms before and after the CA operation are provided in Fig. 7 for the sake of clarity. 


\section{CONCLUSION}

This paper deals with the performance assessment of CA technology, as specified in LTE-Advanced Release 10 and beyond. It is shown that, in the case of intra-band CA, the selection among contiguous and non-contiguous component CA does not influence the performance notably. This is also the case for the inter-band CA of dispersed bands in HetNets when selecting the frequency bands that will be aggregated, as long as that they have the same bandwidth. Thus, hardware complexity, cost, power consumption and other implementation issues should be taken into account in the selection of the most advantageous CA scenario. Besides, CA across cells (multiflow case using frequency bands that belong to different cells of the same RAT, i.e., $\mathrm{CA}(1,5)$ and across licensed and unlicensed bands such as $900 \mathrm{MHz}-5 \mathrm{GHz}$ ) can evolve in many directions beyond the existing specifications. Simply put, a mobile phone data service could be simultaneously carried out by a pico-cell carrier at $5 \mathrm{GHz}$ aggregated with a $\mathrm{CC}$ at $900 \mathrm{MHz}$ belonging to the cellular network, thus implementing the CA over Heterogeneous Bands (HetBand)s.

\section{REFERENCES}

[1] ITU, Requirements related to technical performance for IMT-Advanced radio interface(s), ITU-R Rec. M.2134, Nov. 2008.

[2] J. Thompson, X. Ge, H.-C. Wu, R. Irmer, H. Jiang, G. Fettweis, and S. Alamouti, "5G wireless communication systems: prospects and challenges [guest editorial]," IEEE Commun. Mag., vol. 52, no. 2, pp. 62-64, Feb. 2014.

[3] B. Bangerter, S. Talwar, R. Arefi, and K. Stewart, "Networks and devices for the 5G era," IEEE Commun. Mag., vol. 52, no. 2, pp. 90-96, Feb. 2014.

[4] N. Bhushan, J. Li, D. Malladi, R. Gilmore, D. Brenner, A. Damnjanovic, R. Sukhavasi, C. Patel, and S. Geirhofer, "Network densification: the dominant theme for wireless evolution into 5G," IEEE Commun. Mag., vol. 52, no. 2, pp. 82-89, Feb. 2014.

[5] Z. Shen, A. Papasakellariou, J. Montojo, D. Gerstenberger, and F. Xu, "Overview of 3GPP LTE-advanced carrier aggregation for $4 \mathrm{G}$ wireless communications," IEEE Commun. Mag., vol. 50, no. 2, pp. 122-130, Feb. 2012.

[6] M. Iwamura, K. Etemad, M.-H. Fong, R. Nory, and R. Love, "Carrier aggregation framework in 3GPP LTE-advanced," IEEE Commun. Mag., vol. 48, no. 8, pp. 60-67, Aug. 2010.
[7] G. Yuan, X. Zhang, W. Wang, and Y. Yang, "Carrier aggregation for LTE-advanced mobile communication systems," IEEE Commun. Mag., vol. 48, no. 2, pp. 88-93, Feb. 2010.

[8] R. Ratasuk, D. Tolli, and A. Ghosh, "Carrier aggregation in LTEadvanced," in Proc. IEEE Vehicular Technology Conference (VTC 2010Spring), Taipei, Taiwan, May 2010.

[9] H. Wang, C. Rosa, and K. Pedersen, "Performance analysis of downlink inter-band carrier aggregation in LTE-advanced," in Proc. IEEE Vehicular Technology Conference (VTC 2011-Fall), San Francisco, California, Sep. 2011.

[10] L. Chen, W. Chen, X. Zhang, and D. Yang, "Analysis and simulation for spectrum aggregation in LTE-advanced system," in Proc. IEEE Vehicular Technology Conference Fall (VTC 2009-Fall), Anchorage, Alaska, Sep. 2009.

[11] K. Pedersen, F. Frederiksen, C. Rosa, H. Nguyen, L. Garcia, and Y. Wang, "Carrier aggregation for LTE-advanced: functionality and performance aspects," IEEE Commun. Mag., vol. 49, no. 6, pp. 89-95, Jun. 2011.

[12] A. Ghosh, R. Ratasuk, B. Mondal, N. Mangalvedhe, and T. Thomas, "LTE-advanced: next-generation wireless broadband technology [Invited Paper]," IEEE Wireless Commun. Mag., vol. 17, no. 3, pp. 10-22, Jun. 2010.

[13] 3GPP Technical Specification Group Radio Access Network, Evolved Universal Terrestrial Radio Access (E-UTRA); Carrier Aggregation; Base Station (BS) radio transmission and reception (Release 10), 3GPP TS 36.808, Rev. 10.1.0, Jul. 2013.

[14] L. Liu, M. Li, J. Zhou, X. She, L. Chen, Y. Sagae, and M. Iwamura, "Component carrier management for carrier aggregation in LTEadvanced system," in Proc. IEEE Vehicular Technology Conference (VTC 2011-Spring), Budapest, Hungary, May 2011.

[15] 3GPP Technical Specification Group Radio Access Network, Evolved Universal Terrestrial Radio Access (E-UTRA) and Evolved Universal Terrestrial Radio Access Network (E-UTRAN); Overall description; Stage 2 (Release 12), 3GPP TS 36.300, Rev. 12.1.0, Mar. 2014

[16] Nomor Research GmbH, LTE-A HetNets using Carrier Aggregation, Newsletter, Jun. 2013.

[17] K. Pedersen, P. Michaelsen, C. Rosa, and S. Barbera, "Mobility enhancements for lte-advanced multilayer networks with inter-site carrier aggregation," IEEE Commun. Mag., vol. 51, no. 5, pp. 64-71, May 2013.

[18] H. Wang, C. Rosa, and K. Pedersen, "Dedicated carrier deployment in heterogeneous networks with inter-site carrier aggregation," in Proc. IEEE Wireless Communications and Networking Conference (WCNC), Shanghai, China, Apr. 2013.

[19] 3GPP Technical Specification Group Radio Access Network, Evolved Universal Terrestrial Radio Access (E-UTRA); Base Station (BS) radio transmission and reception (Release 12), 3GPP TS 36.104, Rev. 12.2.0, Dec. 2013.

[20] V. M. Kapinas, "Optimization and performance evaluation of digital wireless communication systems with multiple transmit and receive antennas," Ph.D. dissertation, Thessaloniki, Greece, Jan. 2014. 